\title{
Solution of Co-evolution Self-adaptive Genetic Algorithm to Production Scheduling Problem of Flexible Manufacturing System
}

\author{
Zou Yun ${ }^{1,2, a}$ and Yin Guo-fü ${ }^{1, b}$ \\ ${ }^{1}$ School of Manufacturing Sci. and Eng., Sichuan Univ., Chengdu 610065, China; \\ ${ }^{2}$ Chengdu Textile College, Chengdu 611731, China. \\ ayz610002@126.com, bgfyin@scu.edu.cn
}

Keywords: Co-evolution; Genetic algorithm; Self-adaptive; Production scheduling.

\begin{abstract}
Improved random selection strategy, self-adaptive crossover and mutation operators, population co-evolution algorithm are adopted in this paper to set up a new genetic algorithm(GA), guaranteeing the universality of population, the thoroughness of solution and the execution efficiency of algorithm in solving multi-process, process crossover, work-piece flow and other condition based scheduling problem of flexible manufacturing system(FMS). The problem of GA in solving FMS scheduling is summarized in this paper, reasons and countermeasures for GA's pre-maturing are studied, longitudinal and transverse comprehensive state-based correlative self-adaptive crossover and mutation operators are raised according to the analysis on GA to avoid local optimization and pre-maturity, the co-evolutionary GA is designed to solve the scheduling model for FMS, and the algorithm is validated for the scheduling of box-type parts for precise machine tools.
\end{abstract}

\section{Overview}

The FMS scheduling problem is further researched constantly with FMS development. Not only work pieces, machine tool, cutter, checking-tool, pallet and other factors, but also disturbance information inside and outside the system must also be integrated into system in the real-time manner in the process of scheduling, researchers conducted researches on FMS scheduling from multiple aspects, and existing scheduling theories were supplemented, perfected and improved while new basic theories were proposed. Researches with variable process paths, diversified objectives, scheduling dynamics and algorithm complex ${ }^{[1]}$ were initiated; constantly improved self-adaptive GA, reactive and other intelligent scheduling strategies were built; processing objects, object grouping, equipment load balance and reasonable processing sequence suitable for FMS and others issues were researched in literatures ${ }^{[2]}$; dynamics with different processing paths and identical processing under the premise that different machine tools are selectable are researched in literatures to conduct machine tool selection and process allocation ${ }^{[3]}$; documentary ${ }^{[4]}$ research changes the sequencing method of the fewest parts in order through effective management on free time of equipment and rearrangement of task sequence; documentary ${ }^{[5]}$ study the way of multi-objective optimization in the production planning and scheduling. Researches on effects of longitudinal and transverse comprehensive state of on scheduling in evolutionary process are seldom though fruitful and comprehensive achieved are made.

This paper is aimed at the demand of FMS dynamic scheduling and the internal and external interference factors to FMS scheduling, in the shortest production time, maximum utilization as the optimization goal, intelligent algorithm is chosen as the main technical means, is presented based on the state of vertical and horizontal to the evolutionarily related adaptive selection, crossover and mutation operators of co-evolution GA, and are studied the FMS production intelligent scheduling problem based on multi-procedure, crossing process, the work-piece flow combination and so on. 


\section{Self-adaptive Co-evolutionary GA to Solve FMS Scheduling Problem}

2.1 Reasons and Countermeasures for GA Premature.GA serves as the model to a simulate natural selection and genetic mechanism of Darwin's biological evolutionism, it is earlier applied in research in the field of production scheduling, and substantial corresponding research achievements are available, but it is vulnerable to weak local search ability, premature and slow late search as well as significant effects of the population size, probability of selection (Ps), probability of crossover(Pc), and mutation probability $(\mathrm{Pm})$ on the algorithm performance, the two important embodiments of which are "population diversity" and "selective pressure". Extra selection pressure can accelerate the rate of algorithm convergence, but it can make individuals in a population which are adverse to problem solving "end" rapidly, destroying the diversity of population and having selection and crossover operators loss due effects, thus being trapped in local optimal solution and being "premature". The decrease in selection pressure can increase the probability of search to the global optimal value, but will it reduce the search efficiency.

Furthermore, the crossover operator and mutation operator are actually designed for specific variables, on one hand effects due to inaccurate fitness function can be accelerated, making that some individuals close to global optimal points are individual near local optimal points thereby decreasing opportunities to be selected; on the other hand the evolution of individual close to local extreme points selected are gradually perfected, thereby gradually increasing and approaching local extreme points, further decreasing selected opportunities of newborn individuals close to global optimal points in the evolution and causing the "premature" of populations.

And biological evolution, the evolution of a species contains good genes can individuals in a population rapidly occupy the dominant position, but it does not lose its overall. In GA design, therefore, should be dynamically add new individual strategy, and with selective pressure and the diversity of population, crossover and mutation operators of phase equilibrium mechanism.

In order to realize the requirement of intellectualization, through combined the thought of biological co-evolution, established the cooperative co-evolution GA to solve FMS scheduling problem. Population were randomly divided into several subgroups, various subgroups according to certain mode independently evolved, lateral groups for timely horizontal information sharing and co-evolution, embedded inherited the evolution of the vertical information within population factor, balances the global selectivity and population diversity, suppresses the premature phenomena, at the same time has high global optimization ability and faster convergence speed.

2.2 "Premature" Evaluation Methods for GA Population. Methods evaluating the GA population "premature" include spatial distribution variance method of population individual, entropy method of population, optimum fitness of population, differentials of average fitness of method, etc. However, spatial distribution variance method of population individual reflects the space dispersion degree of individual distribution of population but cannot fully reflect the diversity of population; the entropy method of population, optimal fitness of individuals and differentials of average fitness can't reflect "premature” degree of population individuals in a timely manner.

Researches show that the fitness of individuals varies with different population distributions, hence the dispersion degree of population fitness distribution can be utilized to demonstrate the diversity of individual distribution within the population.

Suppose that a population is comprised by individual $X^{1}, X^{2}, \cdots, X^{i}, \cdots, X^{M}$ (M denotes chromosome number in each population), and the fitness of each individual represents $f^{1}, f^{2}, \cdots, f^{i}, \cdots, f^{M}$ respectively. Therefore:

$$
\begin{aligned}
& f_{\min }=\min \left(f^{1}, f^{2}, \cdots, f^{M}\right) \\
& f_{\max }=\max \left(f^{1}, f^{2}, \cdots, f^{M}\right) \\
& f^{\prime i}=\frac{f^{i}-f_{\min }}{f_{\max }}
\end{aligned}
$$




$$
\begin{aligned}
& D N^{i}=\left.\operatorname{Count}\left(f^{\prime 1}, f^{\prime 2}, \cdots, f^{\prime M}\right)\right|_{l_{i-1}} ^{l_{i}} \\
& D N_{\max }=\max \left(D N^{1}, D N^{2}, \cdots, D N^{M}\right) \\
& D D^{i}=\frac{D N^{i}}{D N_{\max }}
\end{aligned}
$$

Where: $D N^{i}$ represent individual number within interval, $\left.\right|_{l_{i-1}} ^{l_{i}}$ denotes measuring range, $D D^{i}$ signifies the dispersion.

2.3 Solution of Co-evolutionary GA to Algorithm and Model for FMS Scheduling. According to the discussion in 2.1, the sub-population selects the quantity in the operation process by use of the excellent individual library senders, achieves information sharing between populations and advantages complementing, that is to introduce optimal individuals in $\mathrm{n}$ general populations to replace inferior individuals in the population; dynamic replacement method is utilized to realize longitudinal information sharing and advantages complementing, after completion of each ordinary population co-evolution, excellent individuals in the population are elected to excellent individuals library according to the fitness value; individuals of excellent individual library supplement individuals lacked each population evolution before each population evolution, forming a new evolutionary population, thereby forming the mechanism that vertical and horizontal co-evolutions mechanism implement inter-population information sharing and advantages complementing.

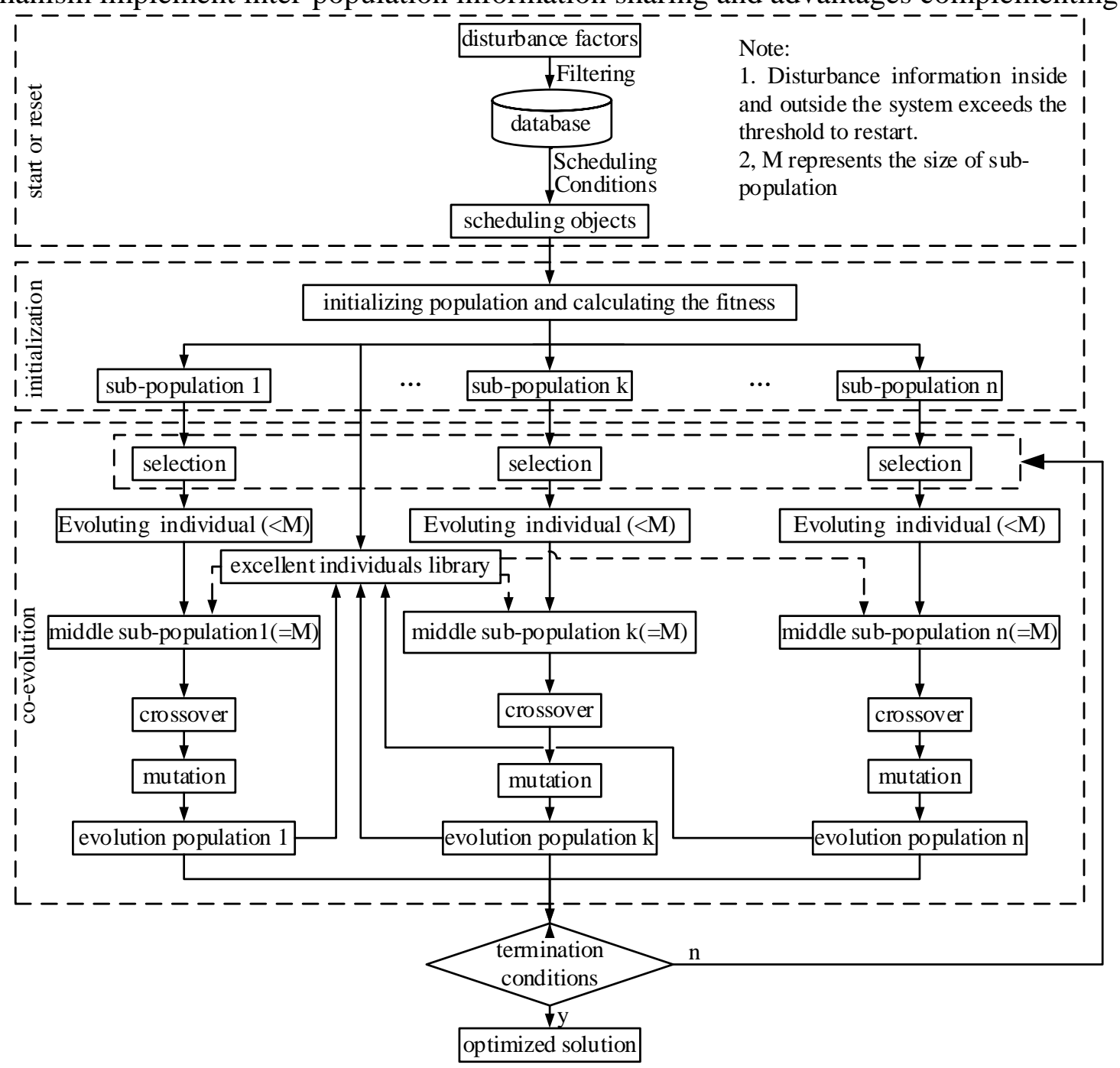

Fig. 1 Solution of Co-evolutionary GA to FMS Scheduling Problem 


\section{Key Methods}

\subsection{Algorithm Design.}

(1) Coding

Real number coding is applied to be the coding scheme, each serial number represents a process of work pieces to express evolutionary process through the change in serial number location, and each serial number consists of the information for the state of processed object, working process and equipment required.

(2) Logic Description to Multi-objective Scheduling

Following algorithm steps are adopted according to the scheduling for single goal or multiple goals selected:

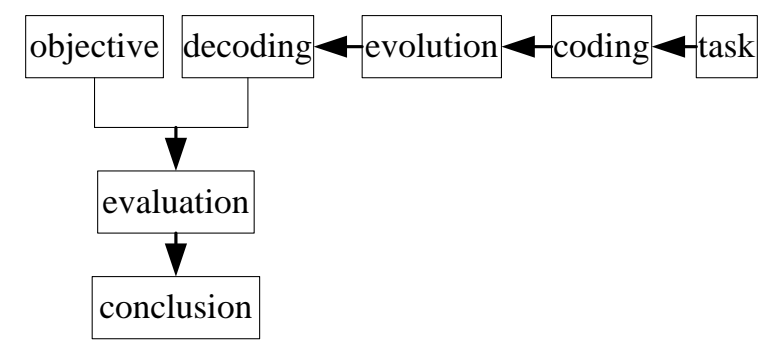

Fig. 2 Logical Descriptions to Targets

(3) Design of Self-adaptive Genetic Operators

1) Selection Operator Design

I Survived expectations of individual $\mathrm{X}^{\mathrm{i}}$ in the number of next generation of populations:

$$
N_{x}^{i}=\frac{M \times f^{i}}{\sum_{i=1}^{M} f^{i}}
$$

II The number of survival of each individual in next generation is $\left[N_{x}^{i}\right]$, the total number of next generation of individuals is $\sum_{i=1}^{M}\left[N_{x}^{i}\right]$.

III The number of next generation of undetermined individuals is $M-\sum_{i=1}^{M}\left[N_{X}^{i}\right]$, and they are supplemented through the high quality library of individuals.

2) Design of Crossover Operator and Mutation Operator

The algorithm converge towards global optimal and restrain premature is the highlight of research. The probability of crossover (Pc) and the probability of mutation (Pm) are self-adaptively and dynamically changed according to the size of the fitness value and distribution characteristics to avoid the shortcomings of being random searching by larger ,being difficulty to deviate local extreme points, and complete global optimization within a relatively short period of time.

Values of the Pc and Pm are determined by virtue of the change in favg-fmax and dispersion degree of population, the entire population tends to converge when favg-fmax is lessened, Pc and $\mathrm{Pm}$ increase, and vice versa; the entire population runs into local optimization when the population dispersion is lessened, Pc and Pm increase, otherwise Pm and Pc. Pc and Pm decreases, the self-adaptive formula for Pc and Pm are as follows:

$$
P_{c}= \begin{cases}k_{1} & \mathrm{f}<\mathrm{f}_{\text {avg }} \\ \mathrm{k}_{2}\left(\frac{\mathrm{f}_{\text {max }}-\mathrm{f}}{\mathrm{f}_{\text {max }}-\mathrm{f}_{\text {avg }}}\right) D D_{t}^{i} & \mathrm{f} \geq \mathrm{f}_{\text {avg }}\end{cases}
$$




$$
P_{m}= \begin{cases}k_{3} & \mathrm{f}^{\prime}<\mathrm{f}_{\text {avg }} \\ \mathrm{k}_{4}\left(\frac{\mathrm{f}_{\text {max }}-\mathrm{f}^{\prime}}{\mathrm{f}_{\text {max }}-\mathrm{f}_{\text {avg }}}\right) D D_{t}^{i} & \mathrm{f}^{\prime} \geq \mathrm{f}_{\text {avg }}\end{cases}
$$

Module

$\mathrm{k} 1, \mathrm{k} 2$ : Crossover control parameters, $k_{1}, k_{2} \in(0,1)$.

$\mathrm{k} 3$,k4: Mutation control parameters, $k_{3}, k_{4} \in(0,1)$.

$\mathrm{f}$ : Larger fitness value of two individuals mutually crossover.

favg: Average fitness value of each sub-population.

$\mathrm{f}^{\prime}$ : Fitness values of individuals to be mutated.

3.2 Handling of Complex Process. The machining of work pieces in FMS production process, especially the machining of precise machine tool boxes needs to be processed on different machine tools in accordance with certain procedures, the following “deadlock” phenomenon may appear:

$$
\begin{aligned}
& M_{k} M_{k} 1 \rightarrow M_{k} \stackrel{(p t, i+1)}{\longrightarrow} M_{k} 3 \rightarrow \stackrel{(p s, j)}{\rightarrow} M_{k}^{4} \rightarrow M_{k} 5 \cdots M_{k} k^{\prime}
\end{aligned}
$$

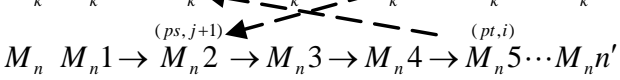

$(p t, i+1)$

$M_{k} 2$ indicates that scheduled machining at the 2nd position of the kth machine is the $\mathrm{i}+1$ th procedure of work piece, and others are ditto.

The unlocking is realized through the translation of genes. It turns to be Formula (11) upon unlocking.

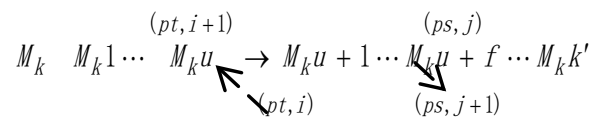

$$
\begin{aligned}
& M_{n} \quad M_{n} 1 \ldots M_{n^{\prime}} V M_{n^{\prime}} V+f^{\prime} \rightarrow M_{n^{V}}+f^{\prime}+1 \cdots M_{n} n^{\prime}
\end{aligned}
$$

Meanwhile, the allocation cost of machining center tools is expensive, the coordination between the pallet and machine tool imposes customization requirements in the processing of precise machine tool box, hence the requirement of tools or pallets replacement exist during the operation is imposed. The operation of part and tool flows needs to consume effective time of transport device and system, hence the object replacement of formulas (12) (14) is determined.

$$
\begin{aligned}
& t_{d}=\sum_{i=1}^{n}\left(t_{d l}(i)+t_{d q}(i)\right) \\
& t_{p}=\sum_{i=1}^{n}\left(t_{p l}(i)+t_{p q}(i)\right) \\
& t_{j}= \begin{cases}t_{d} & \mathrm{t}_{\mathrm{d}}<\mathrm{t}_{\mathrm{p}} \\
t_{p} & \mathrm{t}_{\mathrm{d}} \geq \mathrm{t}_{\mathrm{p}}\end{cases}
\end{aligned}
$$

Where $t_{d l}(i)$ signifies the loading and unloading time to replace the ith tool, $t_{d q}(i)$ represents transport time of the ith tool, $t_{p l}(i)$ denotes the time of loading and unloading for the pallet, and $\mathrm{w}$ denotes the $t_{p q}(i)$ transport time of the pallet.

3.3 Optimized Objectives and Evaluation. Scheduling schemes can be chosen in MFS production scheduling according to different optimized objectives, and there are substantial optimized objectives: Including shortest lead time, shortest delivery time, shortest production time, 
most equipment utilization, shortest time of machine occupation and shortest processing time of remaining processes, fewest inventory and minimum remaining processes. These formulas for optimized objectives are shown in Table 1.

Table1 Fitness Evaluation

\begin{tabular}{|c|c|}
\hline Objective & Formula \\
\hline Shortest time consumption for production & $u_{1 j}=\min _{1 \leq j \leq m} \max _{1 \leq k \leq n} T_{j k}$ \\
\hline Shortest time consumption for machine occupation & $u_{2 j}=\min _{1 \leq j \leq m} \sum_{1 \leq k \leq n}^{n} T_{j k}$ \\
\hline$\ldots \ldots$ & $\ldots \ldots$ \\
\hline Maximum rate of equipment utilization & $u_{s j}=\max _{1 \leq j \leq m}\left(\left(\sum_{s=1}^{p} t_{s}\right) / \sum_{k=1}^{n} T_{j k}\right)$ \\
\hline Comprehensive evaluation & $u=\max _{1 \leq j \leq m}\left(\sum_{i=1}^{s} o_{i} \times w_{i} \times \frac{u_{i j}}{\sqrt{\sum_{k=1}^{m} u_{i k}^{2}}}\right)$ \\
\hline \multicolumn{2}{|c|}{$\begin{array}{l}\text { Notes: } \\
T_{j k}: \text { Hours serviced of the } k^{\text {th }} \text { machine tool in the } j^{\text {th }} \text { group of chromosomes } \\
\mathrm{s}: \text { Total number of optimized objective } \\
t_{\mathrm{s}}: \text { Process time of work pieces on machine tools } \\
\mathrm{p}: \text { Number of work pieces } \\
\mathrm{n}: \text { Number of machine tools } \\
\mathrm{m}: \text { Chromosome number } \\
\mathrm{w}_{\mathrm{i}}: \text { Weight coefficient } \\
\mathrm{o}_{\mathrm{i}}: \mathrm{o}_{\mathrm{i}} \in\{0,1\} \text {, The value will be represented with } \mathrm{o}_{\mathrm{i}}=1 \text { when the objective is selected, or it wil } \\
\text { be presented with } \mathrm{o}_{\mathrm{i}}=0\end{array}$} \\
\hline
\end{tabular}

\section{Algorithm Application}

4.1 System Structure and Requirements. The system composition includes five sets of double-worktable CNC machining centers, one washing machine, one measuring machine, one setting station, one control center, a rail guided vehicle and guidance device, a pallet library, 36 sets of pallet and other auxiliary equipment and laid out into three rows of parallel straight lines as well as the tool of library and other related ancillary facilities.
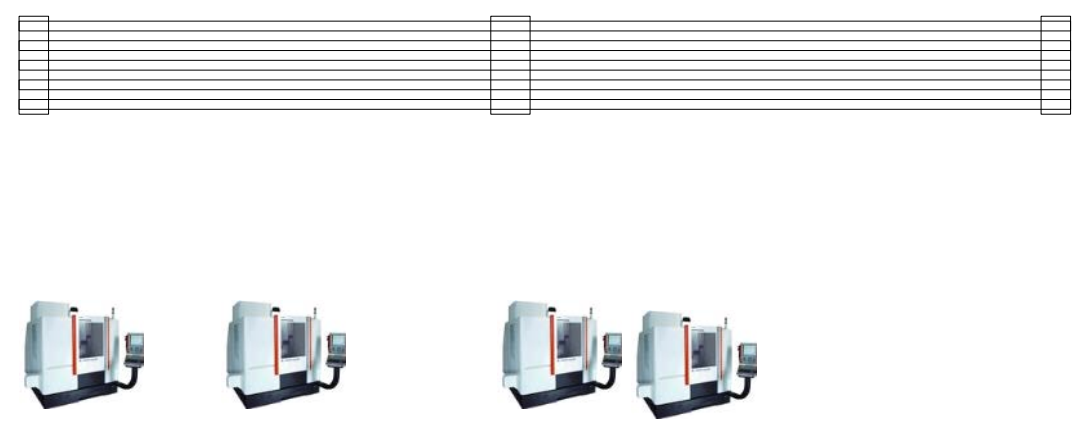

\section{Fig. 3 layout of FMS}

Operating Principle: The setting station completes the loading and unloading part on a pallet, the vehicle, as the connecting unit of the system, complete the conveying of work pieces between 
working stations, the machining center completes the machining of work pieces, empty pallets and work piece pallets are stored in the library of pallets, the cleaning machine takes cleaning on work piece during and after the machining process, measuring machine tests work pieces, the control center completes the process control on the vehicle and machining center and so on.

Major Time Elements: Travel time of vehicle between devices, the time for loading, unloading, processing, cleaning and measuring and other auxiliary time.

Scheduling Requirements: Confirm the time point for the beginning and ending of each task.

4.2 Running Logistics Process of FMS. IN FMS logistics, the vehicle is center, the work pieces is base, the machining centers, measuring machine and cleaning machine is scheduling objects, the needing time of machine tools and vehicle exits time conflict. The basic process is: loading processing - cleaning - testing - unloading.

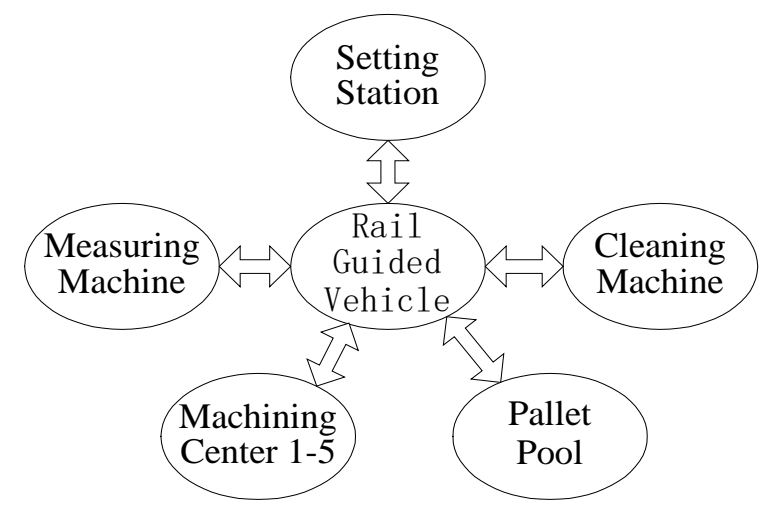

Fig.4 Work-piece flow's routes in FMS

The basic relationship is shown in figure 4 .

Major processes for traveling are as follows:

(1) Preparation

Preparation of Work Piece: Work Piece and Pallet $\rightarrow$ Setting Station $\rightarrow$ Loaded Work Piece on Pallet $\rightarrow$ Vehicle- Pallet Library or Machine Tool.

Preparation of NC Programs and Tools

(2) Processing

Vehicle Arriving Library of Pallets $\rightarrow$ Delivery Pallet with Work Piece $\rightarrow$ Putting Pallet on Machine Table $\rightarrow$ Exchange Position of Table $\rightarrow$ Process.

Vehicle Arriving Machine Tool $\rightarrow$ Lift Pallet on Work Table $\rightarrow$ Library of Pallets, other Machine Tools, Setting station.

(3) Quit:

Vehicle Arriving Machine tool or Library of Pallets $\rightarrow$ Lift Processed Work-piece $\rightarrow$ Delivery to Setting station.

The working process for other devices (e.g: The cleaning machine, measuring machine, and Setting station) is the same to the transport process of work pieces.

4.3 Conditions for FMS Dynamic Scheduling of Production. The real-time dynamic scheduling for FMS dynamically develops logistics planning based on real-time processing state of system, available features of resources and real-time optimization objectives, realizing dynamic sequencings of work pieces and dynamic allocation of resources.

(1) Most of work pieces are single piece or in small mount, and determine the availability of grouping approval based on system statement and integrated optimization objectives of FMS, and develop work pieces and processing sequence.

(2) Formulate work pieces processing sequence according to the state of FMS system and optimization goal when multiple-task queue waits for implementations on a sets of processing equipment. 
(3) Determine the implementation order of tasks according to the state of the system and the optimization goal under the condition that sets of machining equipment are in common model tasks.

(4) Develop the execution order of work tasks in real-time manner under conditions of resource redistribution and comprehensive reconstruction of tasks.

(5) Develop the execution order of work tasks in timely manner under the condition of comprehensive reconstruction of resources and tasks.

The resource, work task and the execution state possessing the randomness, enabling work pieces to be process access into FMS in uncertain time, meanwhile completed work pieces leave FMS in uncertain time. Therefore, FMS may have differentially excessive threshold and may exist in planed and actual statuses at any time point, the occurrence point of the event also serves as the decision point of rescheduling, real-time scheduling system at each time point may have different decision contents, and scheduling rules-based reselection is require for the equipment, work piece and tool and process path, time distribution, etc.

In research process, the changing states of different groupings and different evolution processes in the course of achieving an optimization are as follows:

Table 2 Utilization State for FMS Production Scheduling Equipment

\begin{tabular}{|c|c|c|c|c|c|}
\hline \multirow{2}{*}{ Item } & \multicolumn{5}{|c|}{ Utilization Rate of MC (\%) } \\
\cline { 2 - 6 } & MC 1 & MC 2 & MC 3 & MC 4 & MC 5 \\
\hline 2nd sets & 70.2 & 73.5 & 75.6 & 80.1 & 80.5 \\
\hline 4th sets & 71.9 & 75.1 & 77.4 & 81.1 & 81.5 \\
\hline 6th sets & 73.2 & 76.1 & 78.3 & 81.9 & 82.7 \\
\hline 8th sets & 74.1 & 78.1 & 80.6 & 83.7 & 84.5 \\
\hline 10th sets & 73.9 & 78.5 & 80.5 & 83.7 & 84.7 \\
\hline 12th sets & 74.2 & 78.3 & 80.7 & 83.4 & 84.9 \\
\hline
\end{tabular}

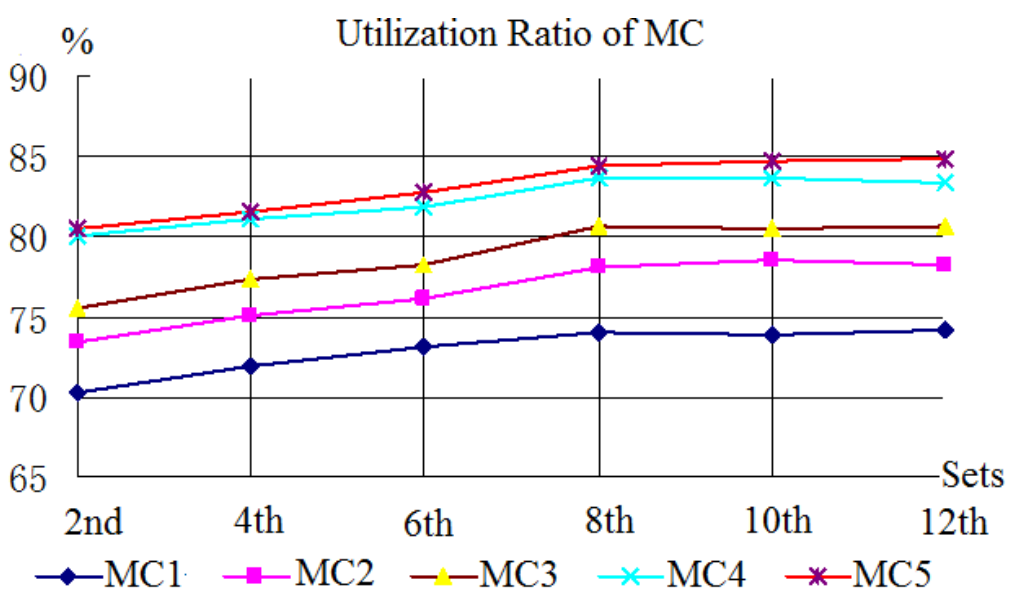

Fig. 5 Effect Tendency of FMS Production Scheduling

The following conclusions can be drawn from Figure 2: The expected targets can be enhanced with the increase in the number of collaborative operation groups, but improved effect on optimization presents no advantage any longer when the number of groups increases to a certain degree, hence intelligent self-learning must be provided in the course of improving operation efficiency and seeking optimal or sub-optimal target expected, independent optimization model should be set up based on historical optimization information of system, and continuous auto-adaptation should be implemented according to changes in the nature of the object . 


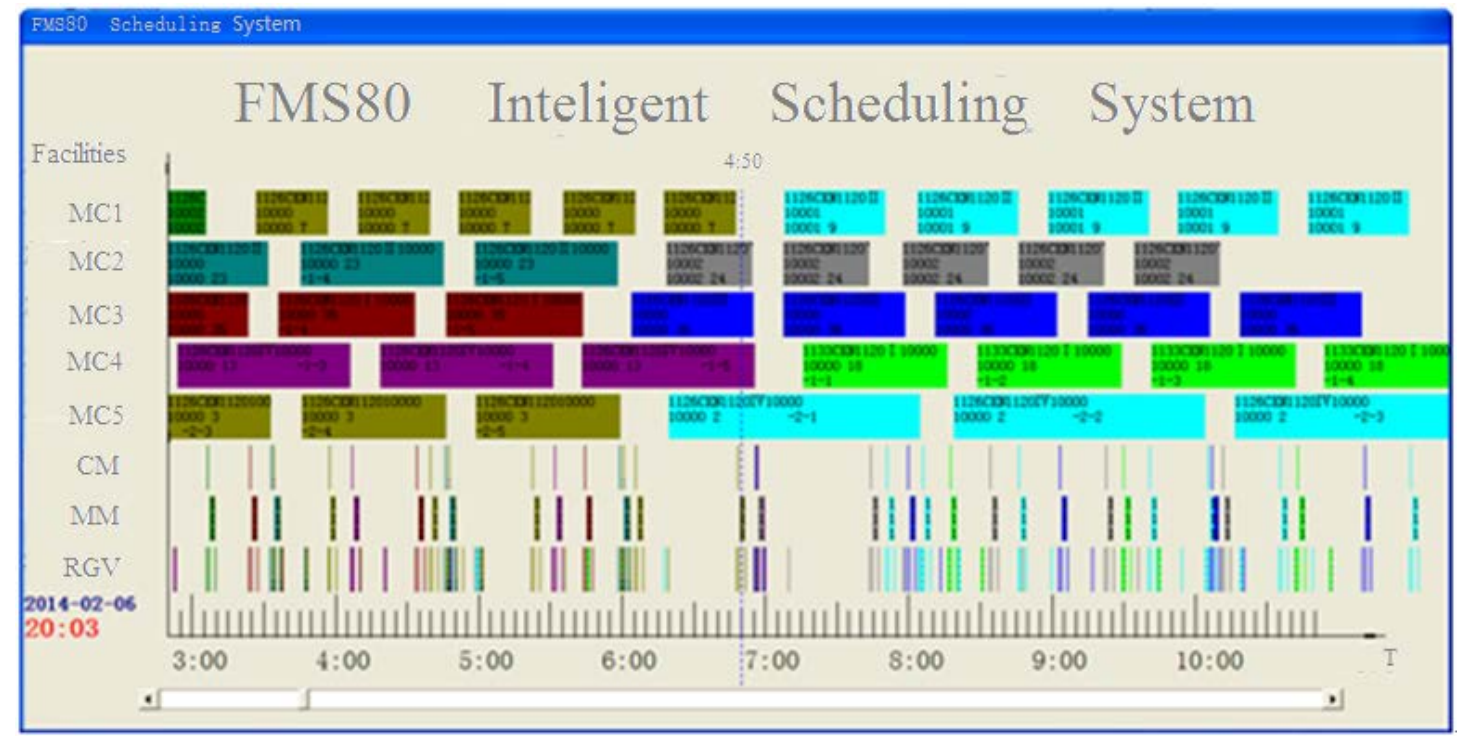

Fig. 6 Intelligent Scheduling Gantt Chart for FMS Production

\section{Conclusion}

Improved self adaptive selection, crossover and mutation operators and co-evolution are utilized in this paper to realize search algorithm of excellent solutions, reflecting the diversity, multihierarchy, self--adaptability and self-organization, realizing global optimization and ensuring the efficiency of optimization, and the convergence rate to obtain globally optimal solution is increased in solving FMS production scheduling problem. The algorithm foundation for can be provided for the flexibility and intelligence with further perfection of mathematical basic theories regarding the data distribution (e.g. disperse model) in genetic evolution process, thereby building highly integrated, dynamic, intelligent, precise and flexible scheduling mechanism, and reducing non value-added time in the process of production.

\section{Acknowledgement}

The research work is supported by special issue of major national science and technology of china (No. 2012ZX04011-031) and Sichuan province science and technology support program (No. 2014GZ0119).

\section{References}

[1] HUANG Fei-hua,LI Si-jie, An Effective Method Using Neural Networks and Genetic Algorithms for FMS Capacity Planning Problems, J. Journal of Systems \& Management, 20 (2011) 496-502.

[2] MA Ding, CHEN Qing-xin, MAO Ning, WANG Zong-zhong, LI Zhan-tao, Parallel machine batch scheduling for due date constraints and setup time, J. Computer Integrated Manufacturing Systems, 18 (2012) 111-117.

[3] M.H.M.A. Jahromi, R. Tavakkoli-Moghaddam, A novel 0-1 linear integer programming model for dynamic machine-tool selection and operation allocation in a flexible manufacturing system, $\mathrm{J}$. Journal of Manufacturing Systems, 31 (2012) 224-231.

[4] Zalmiyah Zakaria, Sanja Petrovic, Genetic algorithms for match-up rescheduling of the flexible manufacturing systems, J. Computers \& Industrial Engineering, 62 (2012) 670-686.

[5] Tadeusz Sawik, Multi-objective due-date setting in a make-to-order environment, J. International Journal of Production Research, 47 (2009) 6025-6231. 